\title{
Incerteza sobre o impacto do capital humano \\ na desigualdade de renda no Brasil
}

Lízia de Figueirêdo*

RESUMO - No Brasil, estudos inter-regionais indicam que as diferenças de anos médios de escolaridade possuem correlação com as desigualdades de renda per capita observadas. Recentemente, a literatura tem se preocupado em medir o capital humano de forma a captar a desigualdade de qualidade na educação. Os resultados indicam pequeno efeito do controle pela qualidade, quando a quantidade de capital humano e/ou dummies regionais já estão consideradas. Realizamos uma contabilidade de nível para os estados brasileiros em 2000 que também conclui ser pequena a diferença entre os resultados anteriores e os corrigidos pela qualidade de ensino.

Palavras-chave: Capital humano. Crescimento econômico. Contabilidade de nível.

\section{INTRODUÇÃO}

Análises das desigualdades de renda per capita (ou de suas taxas de crescimento) entre países revelam a importância da consideração da qualidade da educação na mensuração do capital humano. Segundo Hanushek e Woessmann (2008), a proxy anos médios de escolaridade, usualmente entendida como correspondente ao capital humano de uma região, deixa de ser significativa (ou tem sua importância significativamente reduzida) quando a qualidade da educação é considerada em equações do tipo Barro. Woessmann (2003), por sua vez, observa que a importância relativa do fator capital humano na composição das desigualdades relativas de renda per capita é ampliada para 45\% quando a qualidade da educação é considerada.

O objetivo deste artigo é fazer uma revisão das principais contribuições na literatura brasileira para o estudo do efeito da qualidade de ensino na desigualdade de renda inter-regional no Brasil (Seção 3). Para fins de comparação, iniciamos com uma revisão dos estudos mais relevantes sobre o impacto da quantidade de escolaridade no país (Seção 2). Na quarta seção, uma decomposição de nível para os estados é feita, comparando a importância relativa do capital humano para as desigualdades de renda per capita. São comparadas situações que incluem somente quantidade com especificações que incluem qualidade de educação, medidas através de diferentes proxies.

\footnotetext{
* Doutora em economia pela Universidade de Nottingham. É professora adjunta da Faculdade de Ciências Econômicas da Universidade Federal de Minas Gerais. Endereço eletrônico: lizia@cedeplar.ufmg.br.
} 


\section{QUANTIDADE DO CAPITAL HUMANO E CRESCIMENTO ECONÔMICO}

As principais referências sobre a relação entre anos médios de escolaridade e desigualdade de renda inter-regional no Brasil são: Andrade (1997), Cangussu et al. (2010), Coelho (2007) e Resende (2005). É interessante também observar os resultados da análise com microdados de Sachsida et al. (2004).

Andrade (1997) atualiza o trabalho de Lau et al. (1993) para o período 1970, observando as correlações parciais entre taxa de crescimento do PIB per capita, taxa de crescimento da tecnologia (coeficiente estimado da constante), taxa de crescimento do capital físico (crescimento no consumo de energia elétrica no período), taxa de crescimento da força de trabalho (PEA) e taxa de crescimento do capital humano (taxa de crescimento dos anos médios de escolaridade no período), encontrando um coeficiente de 0,32 para a taxa de crescimento do capital humano. Lau et al. (1993) encontram um coeficiente positivo e significativo para o capital humano para o período 1970-80, de 21\%. A estimação da cross-section é feita por MQO com correção para heterocedasticidade.

Cangussu et al. (2010) testam a adequação relativa entre a especificação de Mankiw, Romer e Weil (MRW) e a de Mincer para estudar os desníveis de renda per capita para 25 estados brasileiros no período 1980-2000. A variável dependente é o nível da renda per capita (ln) e as variáveis independentes são: $\ln$ do crescimento populacional anual, $\ln$ do consumo de energia elétrica, anos médios de escolaridade da população de 25 anos ou mais (Mincer) ou ln desta variável (MRW), além de dummy para tempo. Vários métodos são utilizados na estimação para testar a robustez dos resultados.

$\mathrm{Na}$ equação de MRW, as elasticidades estimadas para a educação variam de $0,4 \%$ - na equação preferida pelos autores (implicando um aumento de $8,5 \%$ na renda per capita para um ano a mais de estudo) - a 1,79\%, enquanto na especificação de Mincer os retornos da educação variam entre 0,071 a 0,394 (15\% de aumento na renda para um ano adicional de estudo na equação preferida). A especificação de Mincer é preferida à de MRW.

Coelho (2006) realiza uma análise cross-section para os municípios brasileiros no período 1970-2000 e encontra clubes de convergência, sendo um dos clubes demarcado pelo valor da taxa de analfabetismo. Também observa a distribuição da renda per capita em 1970 e 2000 através do uso de kernel. O padrão da mesma é bimodal em 2000 e na distribuição ergódica. Analisa então o formato das distribuições de seus componentes: capital físico, capital humano e PTF. Somente o capital humano tem distribuição bimodal na distribuição de 2000 e na ergódica, similar a distribuição da renda per capita.

Em Sachsida et al. (2004), a equação minceriana de salários é estimada em cross-section 
(para 1996) e com dados empilhados (1996-99): a) por MQO; b) pelo método de Garen (primeiro encontra-se um resíduo numa equação de determinação da escolaridade, sendo este utilizado como variável independente na equação de salários); c) com a correção de Heckman. É também estimada através do pseudopainel (criado com cortes de idade) com efeitos fixos. Nos resultados para a equação de salários, o retorno direto da escolaridade varia entre 13\% e 16\%.

Os resultados acima estabelecem indícios fortes da existência de uma relação robusta entre escolaridade e nível de renda per capita. Os trabalhos de Cangussu et al. (2010) e de Sachsida et al. (2004), pela investigação econométrica mais detalhada, nos levam a aceitar que o retorno da escolaridade, tanto micro, quanto macro, se encontre na faixa de 13 a 16\%, um pouco superior ao resultado internacional, e sem fortes indícios de exerternalidades.

\section{O IMPACTO DA QUALIDADE NA DESIGUALDADE DE RENDA}

Nakabashi e Salvato (2007) criam uma nova proxy para qualidade da educação através da soma de três indicadores sobre qualidade de ensino: taxa de aprovação, porcentagem de professores com graduação completa e número de alunos por turma. Com a proxy de qualidade, eles refazem as estimações baseadas no trabalho de MRW para os estados brasileiros. Nas equações de determinação do nível da renda per capita, os anos utilizados são 1970, 1980, 1991 e 2000. A amostra contém 24 estados.

As equações são estimadas por MQO, painel de efeito fixo e randômico. Para os três métodos, são estimadas equações: a) que incluem somente os anos médios de escolaridade; b) que incluem uma variável interativa entre anos médios de escolaridade e o índice de qualidade; c) que incluem anos médios de escolaridade e o índice de escolaridade separadamente.

Nas equações para nível de renda, o coeficiente da variável anos médios de escolaridade é sempre positivo e significativo. Na estimação por MQO, o aumento de 1 ano médio de escolaridade geraria um incremento de $32 \%$ na renda por trabalhador. Na especificação com a variável interativa - índice de qualidade vezes anos médios de escolaridade -, o coeficiente é sempre positivo e significativo. Nas especificações com as duas variáveis (índice de qualidade e anos médios de escolaridade) introduzidas simultaneamente, ambas são positivas e significativas, sendo o impacto dos anos médios de escolaridade menor, de $20 \%$, na renda por trabalhador. Nas estimações por painel, as variáveis de qualidade, quantidade e a variável interativa são positivas e significativas, porém com baixa magnitude.

Soares (2009) usa os dados do IDEB de 2005 para realizar uma contabilidade de nível para a renda per capita municipal, inspirada no trabalho de Woessmann (2003). O PIB per capita (2000) é expresso como uma média ponderada entre o estoque de capital físico (mensurado 
pelo estoque de capital residencial urbano - 2000) e o estoque de capital humano, além da PTF. O capital humano está especificado na forma minceriana, usando anos médios de escolaridade (2000) como proxy de quantidade e o valor médio obtido pelo município no IDEB de 2005. Soares não encontra fortes diferenças com relação à contabilidade original.

Penna (2009) estima a equação de nível similar ao trabalho de MRW para os municípios brasileiros no período 2001-2006 (pooling). Tanto a proxy para qualidade, quanto a proxy de quantidade são positivas e significativas. Há controle por dummies estaduais e pela quantidade de alunos que fizeram a prova (além das variáveis dummies de estado interagidas com o valor do Enem). O impacto de 1 ano adicional de escolaridade seria de 19\% na renda per capita. O impacto do resultado do Enem é significativo, mas de pequena magnitude.

Nos três trabalhos acima, o controle pela qualidade do ensino, ao contrário da literatura internacional, não teve impacto de grande magnitude quando dummies regionais e/ou anos médios de escolaridade já estavam controlados nas especificações.

\section{CONTABILIDADE DE NÍVEL PARA OS ESTADOS BRASILEIROS}

Nesta seção, seguimos a metodologia de Hall e Jones (1998) para decompor o produto per capita em seus componentes - relação capital-produto, capital humano e resíduo. O resíduo para cada unidade federativa (i) é calculado como:

$$
A_{i}=\frac{y_{i}}{h_{i}\left(k_{i} \mid y_{i}\right)^{\frac{\alpha}{1-\alpha}}}
$$

Sendo $A_{i}=$ resíduo da UF; $y_{i}=$ produto per capita da UF; $h_{i}=$ capital humano per capita da UF; $k_{i}=$ capital per capita da UF; $\alpha=0,4$. Os resultados na Tabela 1 mostram a participação de $k / y$ (capa - elevado à potência indicada), A (A) e h (b) no PIB per capita de cada estado, dividido pela mesma relação para o estado de São Paulo (por exemplo, para medirmos a desigualdade regional de renda per capita entre Alagoas e São Paulo, atribuída às diferenças no capital humano, calculamos: $h=\left(b_{A L} / y_{A L}\right) /\left(b_{S P} / y_{S P}\right)$. Os subscritos am, $n s$, idh e $i d e b$ indicam se o capital humano foi calculado utilizando-se somente os anos médios de escolaridade $(a m)$, ham $=\varepsilon^{a m(0,10)}$, ou se houve correção pela qualidade do ensino. Neste caso, utilizamos três medidas de qualidade: o índice de qualidade $(n s)$ de Nakabashi e Salvato (2007), hins $=\varepsilon^{a m[0,10(n s)]}$, o IDH, $h i_{i d h}=\varepsilon^{a m[0,10(i d b)]}$, e o IDEB do ensino médio de $2005, h i_{i d e b}=\varepsilon^{a m[0,10(i d e b)]}$. Esta especificação para o capital humano segue Woeesmann (2003). 
TABELA 1 - CONTABILIDADE DE NÍVEL PARA OS ESTADOS BRASILEIROS

\begin{tabular}{|c|c|c|c|c|c|c|c|c|c|c|}
\hline & $\mathbf{y}$ & capa & $\mathbf{h}_{\mathrm{am}}$ & $\mathbf{h}_{\mathrm{ns}}$ & $\mathbf{h}_{\text {ideb }}$ & $\mathbf{h}_{\mathrm{idh}}$ & $\mathbf{A}_{\mathrm{am}}$ & $A_{n s}$ & $\mathbf{A}_{\text {ideb }}$ & $\mathbf{A}_{\text {idh }}$ \\
\hline$\overline{\mathrm{SP}}$ & 1,000 & 1,000 & 1,000 & 1,000 & 1,000 & 1,000 & 1,000 & 1,000 & 1,000 & 1,000 \\
\hline RJ & 0,959 & 1,061 & 1,027 & 0,910 & 0,968 & 1,015 & 0,894 & 1,008 & 0,948 & 0,904 \\
\hline RS & 0,837 & 0,958 & 0,952 & 0,940 & 0,969 & 0,947 & 0,908 & 0,919 & 0,892 & 0,912 \\
\hline $\mathrm{SC}$ & 0,791 & 1,024 & 0,932 & 0,882 & 0,965 & 0,934 & 0,833 & 0,880 & 0,805 & 0,832 \\
\hline ES & 0,694 & 1,045 & 0,900 & 0,784 & 0,930 & 0,866 & 0,745 & 0,857 & 0,722 & 0,775 \\
\hline PR & 0,690 & 1,061 & 0,909 & 0,904 & 0,909 & 0,888 & 0,726 & 0,730 & 0,726 & 0,744 \\
\hline $\mathrm{AM}$ & 0,672 & 0,542 & 0,960 & 0,636 & 0,775 & 0,883 & 1,107 & 1,671 & 1,372 & 1,204 \\
\hline $\mathrm{MG}$ & 0,594 & 1,110 & 0,876 & 0,806 & 0,903 & 0,848 & 0,627 & 0,681 & 0,608 & 0,647 \\
\hline MS & 0,570 & 0,844 & 0,898 & 0,892 & 0,855 & 0,871 & 0,721 & 0,725 & 0,757 & 0,743 \\
\hline MT & 0,534 & 0,843 & 0,864 & 0,768 & 0,802 & 0,838 & 0,703 & 0,791 & 0,757 & 0,725 \\
\hline $\mathrm{GO}$ & 0,431 & 1,270 & 0,875 & 0,643 & 0,823 & 0,850 & 0,412 & 0,561 & 0,438 & 0,424 \\
\hline $\mathrm{AP}$ & 0,425 & 0,758 & 0,996 & 0,743 & 0,872 & 0,942 & 0,525 & 0,704 & 0,600 & 0,555 \\
\hline RO & 0,392 & 0,852 & 0,911 & 0,715 & 0,853 & 0,857 & 0,485 & 0,618 & 0,518 & 0,516 \\
\hline $\mathrm{BA}$ & 0,370 & 1,038 & 0,766 & 0,572 & 0,706 & 0,716 & 0,469 & 0,628 & 0,509 & 0,501 \\
\hline PE & 0,368 & 1,125 & 0,819 & 0,694 & 0,755 & 0,765 & 0,412 & 0,486 & 0,447 & 0,441 \\
\hline $\mathrm{RR}$ & 0,337 & 1,018 & 0,976 & 0,745 & 0,959 & 0,920 & 0,341 & 0,447 & 0,347 & 0,362 \\
\hline $\mathrm{RN}$ & 0,335 & 1,212 & 0,823 & 0,657 & 0,748 & 0,769 & 0,352 & 0,441 & 0,387 & 0,377 \\
\hline SE & 0,331 & 1,034 & 0,820 & 0,601 & 0,788 & 0,756 & 0,394 & 0,537 & 0,410 & 0,427 \\
\hline $\mathrm{AC}$ & 0,307 & 0,887 & 0,925 & 0,726 & 0,865 & 0,845 & 0,363 & 0,463 & 0,389 & 0,398 \\
\hline $\mathrm{PA}$ & 0,303 & 1,005 & 0,893 & 0,623 & 0,786 & 0,834 & 0,338 & 0,485 & 0,384 & 0,362 \\
\hline $\mathrm{CE}$ & 0,280 & 1,225 & 0,767 & 0,662 & 0,740 & 0,721 & 0,313 & 0,363 & 0,324 & 0,333 \\
\hline PB & 0,269 & 1,135 & 0,798 & 0,668 & 0,740 & 0,730 & 0,307 & 0,367 & 0,331 & 0,335 \\
\hline $\mathrm{AL}$ & 0,249 & 1,232 & 0,756 & 0,583 & 0,706 & 0,695 & 0,282 & 0,365 & 0,302 & 0,307 \\
\hline TO & 0,213 & 1,183 & 0,799 & 0,612 & 0,750 & 0,751 & 0,235 & 0,307 & 0,251 & 0,250 \\
\hline PI & 0,188 & 1,209 & 0,738 & 0,570 & 0,685 & 0,684 & 0,221 & 0,286 & 0,238 & 0,238 \\
\hline MA & 0,163 & 1,211 & 0,743 & 0,565 & 0,675 & 0,681 & 0,190 & 0,250 & 0,209 & 0,207 \\
\hline média & 0,473 & 1,034 & 0,874 & 0,727 & 0,828 & 0,831 & 0,535 & 0,637 & 0,564 & 0,558 \\
\hline média $<30 \%$ & 0,227 & 1,199 & 0,767 & 0,610 & 0,716 & 0,710 & 0,258 & 0,323 & 0,276 & 0,278 \\
\hline
\end{tabular}

FONTE: IPEADATA - os dados para produto, capital físico, população e anos médios de escolaridade são referentes ao ano 2000; Nakabashi e Salvato (2007) para o índice de qualidade; e EDUDATABRASIL, para o IDEB de 2005 do ensino médio.

Na média (exibida na Tabela 1), os estados possuem renda per capita 53\% inferior à de São Paulo. A participação do capital na renda per capita não capta esta diferença (rigorosamente, a participação do capital é 3\% maior do que a de São Paulo). A diferença de participação do capital humano na renda per capita reflete parte da desigualdade de renda: em média, a participação estadual do capital humano na renda é 13\% (27\%) inferior à de São Paulo, quando consideramos somente os anos médios de escolaridade (quando usamos o índice ns). A diferença na participação do resíduo sugere ser a diferença na produtividade total dos fatores a principal responsável pela desigualdade interestadual de renda. Sua contribuição relativa varia de 46\% (com anos médios de escolaridade e IDH) a 36\%, com o índice de ns. O padrão é semelhante para todas as categorias de renda analisadas. Para os estados com menos de 70\% de renda per capita do que São Paulo, a contribuição do capital supera a situação de São Paulo. A menor participação da produtividade total dos fatores na renda é a principal razão que responde pela desigualdade nesse quesito, seguida pela menor participação do capital humano. Podemos também generalizar que quando o capital humano é mensurado somente pelos anos médios de 
escolaridade, as diferenças regionais decorrentes do capital humano são menores. As maiores diferenças são encontradas quando se usa o índice $n s$. No entanto, o uso do IDEB, do IDH ou dos anos médios de escolaridade não afetam significativamente os resultados. O índice de Nakabashi e Salvato (2007) é o que gera maiores diferenças de resultados, aproximando a contribuição do capital humano à da produtividade total dos fatores para mensurar a contribuição para a desigualdade de renda.

\section{CONCLUSÃO}

A principal conclusão a que se chega do ponto de vista da investigação das proxies de capital humano é de que se deve continuar a averiguação sobre a escolha das mesmas. Em princípio, os resultados de testes de conhecimento, como o IDEB, são considerados formas mais adequadas de mensuração da qualidade do ensino. Se isto for confirmado, as diferenças de qualidade entre os estados já seriam bem aproximadas pela diferença de quantidade de ensino. Este resultado reforça o encontrado por Soares (2009).

Se a diferença de quantidade for mais relevante no caso brasileiro, isto pode explicar a pouca robustez e baixa magnitude de impacto do índice de qualidade nas estimativas de Nakabashi e Salvato (2007) e de Penna (2009). Interessante também observar que as diferenças de renda per capita atribuídas ao capital humano, na Tabela 1, com o IDEB, anos médios de escolaridade e com IDH, são as que mais se aproximam dos resultados mais confiáveis das análises econométricas para o Brasil.

Com as evidências até agora, concluímos que as diferenças de capital humano, notadamente de sua quantidade, impactam em torno de 13 a 16\% nas diferenças de renda per capita entre os estados. As diferenças de qualidade não afetam substancialmente os resultados: ou já são bem capturadas pelas diferenças de quantidade, ou ainda não temos uma proxy adequada para qualidade de ensino, que estariam sendo melhor "captadas" pelas dummies regionais. A importância maior do capital humano pode estar circunscrita a alguns municípios atrelados a armadilhas de pobreza, conforme mostra Coelho (2006).

\section{REFERÊNCIAS}

ANDRADE, M. V. Educação e crescimento econômico no Brasil: evidências para os estados brasileiros - 1970/1995. In: XXV ENCONTRO NACIONAL DE ECONOMIA, 1997, Recife. Anais... Brasilia: Anpec, 1998. p. 1529-1548.

CANGUSSU, R. C.; SALVATO, M. A.; NAKABASHI, L. Uma análise do capital humano sobre o nível de renda dos estados brasileiros: MRW versus Mincer. Estudos Econômicos, São Paulo, v. 40, n. 1, p. 153-183, 2010. 
COELHO, R. L. P. Dois ensaios sobre a desigualdade de renda dos municípios brasileiros. Dissertação (Mestrado) - Centro de Desenvolvimento e Planejamento Regional de Minas Gerais, Universidade Federal de Minas Gerais, Belo Horizonte, 2006.

HALL, R. E.; JONES, C. I. Why do some countries produce so much output per worker than others. Quarterly Journal of Economics, v. 114, n. 1, p. 83-116, 1999.

HANUSHEK, E. A.; WOESSMANN, L. The role of cognitive skills in economic development. Journal of Economic Literature, v. 46, n. 3, p. 607-668, 2008.

MANKIW, N. G.; ROMER, D.; WEIL, D. A contribution to the empirics of economic growth. Quarterly Journal of Economics, v. 107, n. 2, p. 407-437, 1992.

NAKABASHI, L.; SALVATO, M. A. Human capital quality in the Brazilian states. Revista EconomiA, v. 8, n. 2, p. 211-229, 2007.

PENNA, R. M. Qualidade educacional e crescimento econômico no Brasil. Monografia (Graduação) - Faculdade de Ciências Econômicas, Universidade Federal de Minas Gerais, Belo Horizonte, 2009.

SACHSIDA, A.; LOUREIRO, P. R. A.; MENDONÇA, M. J. C. Um estudo sobre retorno da escolaridade no Brasil, Revista Brasileira de Economia, v. 58, n. 2, p. 249-265, 2004.

SOARES, C. M. M. Nível de renda e a qualidade da educação nos municípios brasileiros. Monografia PET, Belo Horizonte, 2009.

WOESSMANN, L. Specifying human capital. Journal of Economic Surveys, v. 17, n. 3, p. 239-270, 2003. 
